\title{
Engagements multiples : la rhétorique du GlobalMay Manifesto
}

Multiple Engagement: The Rhetoric of GlobalMay Manifesto

\section{Nana Ariel}

Translator. Maria Brilliant

\section{(2) OpenEdition Journals}

Electronic version

URL: http://journals.openedition.org/aad/1914

DOI: $10.4000 /$ aad. 1914

ISSN: 1565-8961

\section{Publisher}

Université de Tel-Aviv

\section{Electronic reference}

Nana Ariel, « Engagements multiples : la rhétorique du GlobalMay Manifesto », Argumentation et Analyse du Discours [Online], 14 | 2015, Online since 09 April 2015, connection on 23 September 2019. URL http://journals.openedition.org/aad/1914 ; DOI : 10.4000/aad.1914

This text was automatically generated on 23 September 2019.

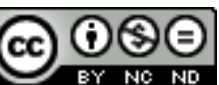

Argumentation \& analyse du discours est mis à disposition selon les termes de la licence Creative Commons Attribution - Pas d'Utilisation Commerciale - Pas de Modification 4.0 International. 


\title{
Engagements multiples : la rhétorique du GlobalMay Manifesto
}

\author{
Multiple Engagement: The Rhetoric of GlobalMay Manifesto
}

\author{
Nana Ariel
}

Translation : Maria Brilliant

\section{Introduction}

1 La fièvre de manifestes qui a submergé le monde depuis les manifestations sociopolitiques de 2011, comparable uniquement au flot d'écriture manifestaire du tournant du $20^{\mathrm{e}}$ siècle ou à celui des années 1960 , a redonné vie à ce genre en tant qu'instrument essentiel de l'engagement civique, incitant ainsi à examiner ses rôles, ses fonctions et configurations au $21^{\mathrm{e}}$ siècle ${ }^{1}$. En dépit d'affirmations réitérées sur la mort du manifeste qui ne serait plus qu'une relique de l'âge des idéologies ${ }^{2}$, et bien qu'il soit souvent utilisé de nos jours sous forme de pastiche (comme dans le titre d'un nouvel arôme de Yves Saint-Laurent : « manifeste »), il est encore pratiqué dans son contexte historique originel, et reste une étape nécessaire dans la formation des mouvements sociaux ${ }^{4}$.

2 Parmi les nombreux textes manifestaires publiés pendant cette vague d'agitations, GlobalMay Manifesto (Mai 2012) ${ }^{5}$, créé par International Occupy Assembly (Assemblée Internationale Occuper) représentant le mouvement mondial Occupy ${ }^{6}$, fut l'un de ceux qui ont été le plus largement distribués, ainsi que les plus vivement critiqués ${ }^{7}$. Face aux affirmations réitérées selon lesquelles Occupy n'aurait pas d'objectifs organisés concrets ${ }^{8}$, les auteurs du manifeste tentent de construire un ethos collectif unifiant, axé sur la protestation contre les tendances contemporaines néolibérales et sur un appel à la démocratisation mondiale dans toutes les sphères sociales et politiques. Afin de mettre en œuvre leur vision dans leur propre pratique, des activistes de divers pays ont coopéré à la rédaction du GlobalMay Manifesto à l'aide d'Internet, et de nombreuses traductions en ont été offertes - bien que généralement accusées de n'être pas suffisamment collaboratives. Certaines critiques ont même rejeté l'idée que le mouvement Occupy puisse avoir un manifeste unifiant - à cause de sa nature 
participative horizontale propagée par un réseau social en ligne, sa répugnance à l'égard de toute hiérarchie et méthode de gestion du haut vers le bas, et son aspiration à se détacher des méthodes politiques traditionnelles. «Occupy parle avec de nombreuses voix mais n'apprécie pas qu'on parle de lui comme d'un collectif », écrivait un critique anonyme (Guest Author, 18-6-2012 ). «Occupy n'a pas besoin d'un manifeste », affirmait un autre (Stuart King, 22-5-2013 ${ }^{10}$ ).

3 Le présent article propose une analyse des mécanismes rhétoriques de GlobalMay Manifesto en se focalisant sur les stratégies utilisées pour construire une collectivité mondiale imaginaire prenant en compte une vaste panoplie d'exigences et de réclamations potentielles. Conscients de la difficulté à pourvoir d'un manifeste un mouvement supposé être "sans manifeste» - un mouvement qui rejette intrinsèquement toute centralisation idéologique -, les auteurs du texte tentent de surmonter cet obstacle en y intégrant une série de voix imaginaires. Au lieu de voir dans la multiplication des voix un simple éparpillement, et de dénoncer le caractère problématique de cette rhétorique manifestaire - comme l'ont fait auparavant aussi bien les activistes que les chercheurs (Gregoratti 2014, O'Sullivan 2014'11), je propose ici de l'examiner comme une tentative de résoudre des problèmes rhétoriques, à savoir de rassembler et concilier des intérêts et des volontés en conflit.

Cette analyse se concentre donc sur le moment délicat de la déclaration elle-même, et sur les modalités selon lesquelles elle est désignée et construite. Je ne chercherai ni à étudier et critiquer la place tenue par les allégations du manifeste dans le débat en cours sur la nature de la démocratie contemporaine, ni à évaluer dans quelle mesure ses rédacteurs ont réussi ou échoué à représenter leur communauté, ou à stimuler le changement. Je considérerai plutôt le manifeste en tant qu'instrument d'agitation sociale en explorant sa tentative complexe de mettre en pratique le «quand dire c'est faire » d'Austin ${ }^{12}$, même s'il ne parvient finalement pas à des résultats au niveau de ses ambitions déclarées, et ne subsiste plus que comme un monument douteux dédié à un mouvement social apparemment fort ébranlé. Sur ce point, je rejoins la suggestion de Martin Puchner: "Que les manifestes individuels accomplissent leurs objectifs ambitieux ou pas [...] importe moins que les stratégies rhétoriques, poétiques, littéraires qu'ils ont utilisées dans le seul but de changer le monde » (2006:2).

Du fait qu'il se veut résolument interdisciplinaire, ce travail articule trois directions théoriques principales. En premier lieu, je m'inspire d'études antérieures sur le genre du manifeste (Abastado 1980, Angenot 1982, Lyon 1999, Burger 2002, Somigli 2003, Puchner 2006, Yanoshevsky 2009, entre autres), afin de cerner et problématiser un héritage générique dont les caractéristiques sont nettement présentes dans le GlobalMay Manifesto. En second lieu, et dans une perspective élargie, je m'appuie sur la démarche fonctionnelle des études consacrées à la rhétorique des mouvements sociaux (Simons 1971, Bowers et al. 2010, Orkibi 2012), qui voient dans les textes déclaratifs et programmatiques non seulement des passeurs d'idées, mais surtout les instruments de mobilisation, d'unification et de construction d'un ethos collectif, mobilisés par des agents sociaux rationnels utilisant des pratiques rhétoriques diverses ${ }^{13}$. Par conséquent, je me servirai également de concepts-clés analytiques et d'outils méthodologiques tirés des études contemporaines, dans le domaine de la rhétorique et de l'analyse du discours (Marcoccia 1994, Amossy 2002). 


\section{La structure hétéroglossique du GlobalMay Manifesto}

6 Le GlobalMay Manifesto est composé de discours multiples et hybrides, créant de ce fait une structure rhétorique peu consistante qui donne l'impression d'un ethos inconsistant et inégal, d'un texte rigide et définitif, alors qu'il se définit lui-même comme un travail en cours. Il utilise un ton autoritaire et résolu en même temps que des expressions marquant l'excuse et l'hésitation, il propose une rupture et un renouveau tout en se réclamant d'une position historique antérieure, il rejette fondamentalement le système socio-politique existant tout en proposant d'y apporter des réformes concrètes, et il tente de créer une collectivité universelle tout en utilisant des tropes et une gestuelle typiques de politiques identitaires.

7 Cette pratique rhétorique d'affrontements et de contradictions, apparemment contreproductive, peut aisément être expliquée comme une tentative de gérer des intérêts conflictuels. Si tout mouvement social tente de concilier des exigences en conflit (Simons $1970: 2-3$ ), dans le cas d'un mouvement mondial qui se targue de représenter une protestation globale de type universel, la variété des perspectives qui s'ouvrent aux partisans potentiels est immense; en conséquence, son potentiel de conflit est extrêmement élevé. Les émetteurs du manifeste désirent représenter aussi bien des activistes qui rêvent de changer le cours de l'histoire, que des modérés qui luttent pour réformer des systèmes existants. Ils aspirent à paraître au service de partisans provenant de pays riches aussi bien que pauvres; ils veulent représenter les anticapitalistes, les environnementalistes, les minorités sexuelles et ethniques et, de fait, tout mouvement social existant, en permettant une cooptation éventuelle. La clé d'une bonne compréhension du GlobalMay Manifesto consiste donc à l'analyser en tant que tentative de représenter un mouvement décentralisé, composé d'auditoires multiples, s'exerçant à diminuer le ressentiment potentiel que peuvent susciter des voix conflictuelles. Cette situation évidemment paradoxale s'exprime par un certain nombre de caractéristiques textuelles et intertextuelles.

En premier lieu, GlobalMay Manifesto a été publié dans des versions différentes dont chacune contient des changements certes mineurs, mais significatifs. Ainsi, une première version publiée sur le site de People's Assemblies Network le 4 mai $2012^{14}$ est légèrement différente de celle qui paraît dans le The Guardian du 11 mai ${ }^{15}$. La seconde version, plus largement diffusée et légèrement plus courte, atténue le ton pompeux de la première. Y disparaît, par exemple, la déclaration dramatique «Nous sommes de retour », et le slogan conventionnel « Liberté, Egalité, Fraternité ». Elle omet également de critiquer le rôle de la presse généraliste dans le maintien et le renforcement de procédures non démocratiques, présentées comme "l'instrument qui fabrique un consensus factice autour de ce système injuste et insoutenable »; cette omission révèle la nécessité de modérer les énonciations conflictuelles à un moment où le manifeste se veut accessible au grand public et appelle le soutien des médias. D'autres versions sur différents sites occultent des parties entières du texte, ne présentant que la déclaration d'ouverture du manifeste, ou encore la liste de ses exigences. Ces changements éditoriaux dissimulés, qui ne sont pas officiellement présentés comme des versions différentes, sont les traces de la négociation complexe et continue à laquelle se livre le manifeste. Ma source de référence principale sera la première version largement diffusée du 4 mai, qui semble avoir subi dans une moindre mesure l'impact de considérations d'édition journalistiques ultérieures ${ }^{16}$ - ce qui n'implique nullement 
qu'il s'agit là d'une " version originale " prétendument plus authentique que d'autres. Il faut plutôt la considérer comme l'un des premiers maillons d'une chaîne de modifications jamais achevée, donnant ainsi à voir le manifeste comme un texte ouvert qui « se produit » dans l'intervalle créé par des versions diverses, où la négociation sur les formulations et le style apparaissent au grand jour, et où des tensions, des heurts et des contradictions se font sentir.

Deuxièmement, le heurt des voix est également apparent dans la structure inhérente au texte. Le manifeste peut être divisé en quatre parties, dont chacune semble adopter un schème discursif totalement différent, comme on le verra dans la suite :

- a. une déclaration générale qui met en scène une rhétorique manifestaire autoritaire typique, activée par le pathos,

- b. une partie apologétique qui diverge complètement du ton décisif de la première (dans certaines versions, elle est même stylisée par une couleur et des caractères typographiques différents),

- c. une longue liste d'exigences comprenant trois sections principales et vingt-neuf soussections, exposant un programme spécifique et concret portant sur un éventail de problèmes, et qui semble manquer de toute logique susceptible de lui conférer quelque organisation,

- d. un appel à l'action conventionnel de type anaphorique : « Ceci est un Printemps Universel, nous serons présents en Mai 2012, nous lutterons jusqu'à la victoire. Nous ne cesserons pas d'être des êtres humains. Nous ne sommes pas des chiffres. Nous sommes des hommes et des femmes libres. Pour un printemps mondial! Pour une démocratie mondiale et pour la justice sociale! Descendez dans les rues en mai 2012 ! ${ }^{17}$ »

Le langage et la structure hybrides du texte impliquent une oscillation constante entre diverses tendances et méthodes d'activisme. Je vais à présent localiser et analyser les différents « dialectes » et pratiques discursives de ce manifeste, dans le but d'explorer son hétéroglossie immanente. J'étudierai tout d'abord le modèle performatif que nous offre le manifeste qui oscille entre celui du document rigide, définitif, et celui du travail en cours marqué par sa flexibilité. J'élargirai ensuite le débat en examinant l'oscillation du manifeste entre un discours de révolution et un discours de réforme. Finalement, j'analyserai deux discours populaires particulièrement dominants dans ce manifeste : celui des politiques identitaires, et le discours écologique. La prépondérance remarquable de ces discours, qui se donnent souvent à voir dans le manifeste comme des vérités évidentes, révèle leur rôle dans la doxa de l'activisme contemporain, c'est-àdire sa base commune partagée et incontestée, et les connaissances communes qui s'imposent comme des évidences ${ }^{18}$.

\section{Le Manifeste mondial constitutif ultime}

11 Par sa structure générale et son ton dominant, GlobalMay Manifesto exprime le désir d'imaginer ce que serait le manifeste révolutionnaire mondial ultime, et de produire un texte prototypique qui fonctionnerait comme «le » manifeste - un document officiel représentatif d'une vague mondiale de manifestations et, dans ce sens, un texte constitutif rigide. C'est dans cette perspective que sa déclaration initiale adopte des caractéristiques manifestaires conventionnelles, et construit un discours de crise et de rupture. 
Nous vivons dans un monde contrôlé par des forces incapables de conférer liberté et dignité à la population du globe (si même elles en avaient jamais été capables). Un monde où l'on nous dit « on n'a pas le choix » devant la perte des droits gagnés par les longues et dures luttes de nos ancêtres. [...]

Mais nous ne nous sommes pas tus! Notre conscience s'est éveillée, et nous nous sommes unis à la vague de conscience collective qui répand maintenant la lumière et l'espoir à tous les coins du monde. De la Tunisie au Square Tahrir, de Madrid à Reykjavik, de New-York à Bruxelles, des peuples se soulèvent. Dans le Printemps arabe, dans la dignité de l'Islande, dans la rage empreinte de dignité du $15 \mathrm{M}$ et de Occupy Wall Street. Ensemble, nous avons dénoncé le statu quo. Nos efforts le clament clairement «Assez !» [...]

C'est pourquoi nous, femmes et hommes, habitants de cette planète, nous unissons à nouveau pour faire entendre nos voix ce 12 mai. Tout autour du monde. [...]

Ici et maintenant, nous sommes de retour. Nous nous sommes réveillés, et pas seulement pour nous plaindre ! [...] Pour dire les choses simplement, nous voulons un monde régi par les valeurs de liberté, d'égalité et de fraternité - le vieux rêve de nos ancêtres lorsqu'ils se sont élevés contre l'oppression, dans les générations précédentes, sur la planète tout entière!

L'affirmation initiale que "Nous vivons dans un monde contrôlé par des forces incapables de conférer liberté et dignité à la population du globe ", avec la qualification rhétorique entre parenthèses " (si même elles en avaient jamais été capables) » absent des versions ultérieures, montre la tentative de construire une histoire partagée imaginaire, qui conduira inévitablement à une situation révolutionnaire. En soulignant une condition intolérable marquée par une absence universelle de liberté et de dignité, le manifeste bâtit un mythe de rupture ${ }^{19}$, en utilisant les actes de parole typiques de l'urgence, du réveil, de la rage et du soulèvement, avec des points d'exclamation passionnés à valeur de mobilisation - «nous ne nous sommes pas tus!»; « Nos efforts le clament clairement: "assez!" »; "Nous nous sommes réveillés!20» La structure rhétorique de la partie initiale peut donc être décrite en termes d'" arguments par le signe »: elle met en œuvre des tropes conventionnels de crise et d'engagement qui rattachent le texte à une tradition révolutionnaire, en exploitant le capital symbolique d'un discours révolutionnaire préétabli. Elle offre l'activation familière d'un certain esprit de rébellion et de résistance, comme si elle déclarait «Si ce que je dis sonne comme un manifeste révolutionnaire, alors je suis un manifeste révolutionnaire ».

De plus, non seulement le manifeste résonne comme un discours révolutionnaire, il est aussi expressément présenté comme le retour à un ancien combat révolutionnaire latent. Cette position est exprimée de manière paradigmatique, dans l'annonce des émetteurs du manifeste - «nous sommes de retour». A travers le discours révolutionnaire, ils communiquent aussi un sentiment fort de répétition et de réitération. Déclarant qu'ils s'unissent "une nouvelle fois ", afin de faire revivre les combats de leurs "ancêtres", ils bâtissent un lien généalogique entre les luttes révolutionnaires historiques et leur propre révolte.

La source de cette généalogie est implicitement représentée par l'événement constitutif de la modernité et le modèle archétypique incontesté de l'engagement civique - la Révolution française - lorsque les auteurs déclarent : « nous voulons un monde régi par les valeurs de liberté, d'égalité et de fraternité - le vieux rêve de nos ancêtres lorsqu'ils se sont élevés contre l'oppression, dans les générations précédentes, sur la planète tout entière! ". Cependant, les expressions de type extrêmement général comme "générations précédentes" et "sur la planète tout entière " indiquent que la mobilisation de la Révolution française ne se réfère pas à un événement historique 
spécifique, ni ne se réclame d'un mythe concret; elles constituent plutôt un trait conventionnel du discours révolutionnaire. Ce mode d'expression montre bien que la Révolution française est largement utilisée dans le discours populaire contemporain en tant que "meme » - une unité sémantique reproduite et détachée de son origine ou de son contexte, répandue par des imitations récurrentes ${ }^{21}$. Le meme de la "Révolution française " inclut souvent la répétition de certains éléments (auxquels on peut se référer en tant que memes secondaires), tels la formule "liberté, égalité, fraternité ", des utilisations imprécises du terme "république ", un format de déclaration des droits de l'homme, une "assemblée du peuple ", ainsi que des images emblématiques telles que la guillotine ${ }^{22}$. Ces éléments sont constamment réutilisés - de façon libre - par des blogueurs, des auteurs de manifestes et des manifestants sans relation ni engagement à aucun contexte historique prédéfini. Ils fonctionnent comme des "prêt-à-porter " devenus partie intrinsèque du lexique de la résistance sociale, permettant à de nouveaux agents des luttes sociales d'acquérir rapidement un capital symbolique préliminaire, en se reliant à une tradition révolutionnaire par des allusions conventionnelles, des symboles et des images.

Il se crée ainsi une forte tension entre l'aspiration affirmée à une innovation révolutionnaire totale, et l'utilisation de "prêt-à-porter ", de lieux communs et même de clichés de la révolution. En fait, les curieuses interrelations entre innovation et répétition sont essentielles à toute compréhension du discours révolutionnaire, comme le terme même de révolution, dans son sens copernicien, signifie répétition ${ }^{23}$; les auteurs de la Révolution française eux-mêmes considéraient leur action comme un retour à une époque antérieure (Puchner 2006: 259). La dynamique révolutionrépétition reflétée dans les manifestes est particulièrement importante pour bien comprendre la conception personnelle de ceux qui font aujourd'hui entendre une voix protestataire (ibid., 259-62), et elle apparaît de manière très nette dans la rhétorique du GlobalMay Manifesto. Ainsi, deux concepts distincts de la révolution - "révolution comme innovation" et "révolution comme répétition" - sont simultanément accentués tout au long du manifeste. Cette dualité est clairement exprimée vers la fin du texte, lorsque ses auteurs déclarent :

Certains d'entre nous pensent qu'une nouvelle Déclaration Universelle des Droits de l'Homme, convenant au $21^{\mathrm{e}}$ siècle, écrite de manière participative, directe et démocratique, devrait être élaborée. Mais tant que la Déclaration des Droits de l'Homme actuelle définit nos droits, elle doit être appliquée chez tous - aussi bien dans les pays riches que pauvres.

L'adoption de la Déclaration française comme modèle politique général à suivre et, simultanément, son rejet en tant qu'instrument politique de facto, illustre l'ambivalence du manifeste envers la situation révolutionnaire. Est-ce qu'être "révolutionnaire " signifie faire partie d'une tradition révolutionnaire en imitant des "prêt-à-porter " révolutionnaires préexistants? S'agit-il d'une tentative pour actualiser des motifs révolutionnaires dans de nouvelles conditions? Ou bien devrait-ce être, comme le suggèrent certains critiques du manifeste, l'abandon de toute méthode politique familière telle qu'une Déclaration de droits unificatrice, pour favoriser une approche totalement inventive? Les auteurs du manifeste essaient d'ouvrir cette question au débat. La suggestion d'une réécriture potentielle future d'une nouvelle Déclaration des Droits de l'Homme implique que le manifeste est une œuvre en cours négociable qui se poursuit au fil du temps, plutôt qu'un document révolutionnaire constitutif et définitif. 


\section{Un travail en cours de type collaboratif}

collaborateurs habituels du mouvement, cette partie offre une apologie et une justification de l'apparente incohérence du manifeste. En déclarant que le processus de gestation du manifeste était ardu et a nécessité de nombreux compromis, ses auteurs recherchent l'estime et l'empathie des destinataires. Pour les participants qui ont pris part à la formulation du manifeste, ainsi que pour ses nouveaux collaborateurs potentiels, cet extrait revêt une signification supplémentaire: il vise à écarter à l'avance les critiques potentielles du modèle participatif qui avait présidé à sa création, repoussant d'éventuels ressentiments, construisant un ethos partagé inclusif, et appelant à un dialogue en ligne. On peut noter d'ailleurs que dans la version publiée sur le site People's Assemblies Network, cet extrait était souligné graphiquement, attirant ainsi beaucoup plus l'attention que dans la version publique du Guardian; cette différence implique qu'il est plus pertinent pour le discours interne des activistes que pour le discours public général. De ce fait, la rhétorique non conclusive s'avère plus bénéfique que nuisible - bien qu'elle paraisse polarisante, elle renforce en fait un sentiment de la collectivité bâti sur un modèle communicationnel plutôt que sur un assortiment d'idées prédéterminé.

La position du GlobalMay Manifesto - entre une détermination rigide et une flexibilité inclusive - se dégage clairement d'une brève comparaison avec deux archétypes de manifestes collectifs, qui représentent des modèles contradictoires d'écriture manifestaire - le texte consacré et le travail en continu (work in progress) collaboratif. Le 
premier exemple, celui du texte consacré, est l'archétype du genre : c'est le Manifeste Communiste, qui exerce sans nul doute une grande influence sur le GlobalMay Manifesto,dans la mesure où il constitue un tract socio-politique international. Le manifeste de Marx et Engels a été rédigé, formaté et diffusé comme un texte rigide, fixé, 'sacré' qui ne peut être retouché ni modifié. Depuis sa première publication à Londres en 1848 , les auteurs firent des efforts considérables pour conférer ce statut à leur texte, les seules additions - reflet de contextes et de circonstances variables - étant de nouvelles introductions à de nouvelles éditions, écrites par Marx et Engels euxmêmes (Puchner 2006: 31-38). Bien que conscients de ce que certaines de leurs exigences étaient désormais dépassées, ils insistèrent pour préserver la version originale, en déclarant par exemple, dans leur introduction à l'édition allemande de 1872, que le manifeste était un document historique que ses auteurs eux-mêmes ne se sentaient pas autorisés à transformer, l'intronisant ainsi comme texte sacré (ibid., 35) ${ }^{24}$. C'est cette position qui a été l'un des facteurs prééminents de la canonisation du Manifeste Communiste, et son établissement comme l'un des textes les plus influents et les plus largement diffusés de tous les temps.

21 L'exemple à l'extrême opposé est le second modèle, un manifeste collaboratif qui n'a pas seulement été écrit à notre âge numérique actuel mais a aussi été un pionnier en matière de pensée et de pratique numériques - le Cyberpunk Manifesto, publié en Bulgarie en 1997 par l'artiste cyber Christian As. Kirtchev. Bien que ce manifeste relativement marginal puisse sembler assez éloigné du contexte du présent débat, il représente en fait d'un effort novateur d'écriture manifestaire en ligne et négociable, fonctionnant ainsi indirectement comme un précurseur dans la généalogie du GlobalMay Manifesto. Le mouvement Cyberpunk intégrait des caractéristiques familières de la culture punk en prenant l'Internet comme terrain de jeu; de même que d'autres mouvements numériques, il promouvait une conception du sujet numérique comme entité libre et créatrice qui défie la société institutionnalisée, et qui comporte un potentiel socio-politique révolutionnaire. En conséquence, le Cyberpunk Manifesto a été un texte inhabituellement inclusif, écrit en continu - il a été partagé sur Internet de manière à ce que chaque lecteur puisse activement et immédiatement le reformuler. Il a été ensuite publié en plusieurs versions, présentées dans la terminologie des générations numériques: Cyberpunk Manifesto 2.0 (2003) et Cyberpunk Manifesto 3.0 $(2007)^{25}$.

Ces deux modèles reflètent des stratégies de légitimation différentes. Tandis que le premier se fonde sur la canonisation et la sacralisation qui confèrent au texte une aura d'exclusivité autoritaire, le second reçoit sa légitimation à travers un processus inclusif de collaboration sociale. Le GlobalMay Manifesto se situe quelque part au milieu de cet éventail, entre le texte sacralisé et un travail en continu écrit dans un espace "ouvert", oscillant et hésitant entre les deux pôles. En dépit de son ouverture potentielle due à la possibilité technique de débat collaboratif en ligne, le manifeste n'en exprime pas moins, dans son ton général, le désir de fonctionner comme un document constitutif global finalisé. 


\section{"Une rage empreinte de dignité »: discours de Révolution vs. discours de Réforme} tension mentionnée ci-dessus entre rigidité et flexibilité, est sa fluctuation entre un
discours révolutionnaire et un discours réformateur. Entre la partie apologétique et la liste des exigences, ses auteurs déclarent: "Nos exigences ne sont pas adressées aux gouvernements, municipalités ou membres des parlements - que certains d'entre nous considèrent comme illégitimes, arbitraires ou corrompus. Nous parlons aux peuples du monde, qu'ils fassent ou non partie de nos mouvements. Nous voulons un autre monde, et un tel monde est possible». Par cette déclaration, les auteurs semblent clairement définir leurs auditoires : les peuples plutôt que les institutions. Ils partent du principe que des révolutions ne se font, et qu'un « autre monde » ne peut se réaliser, que par «le peuple » dégagé de toute sorte de puissance institutionnelle. Toutefois, à l'inverse de cette déclaration initiale, la liste des exigences qui suit se réfère presque entièrement à des réformes institutionnelles. Pensions de retraite, règlementation des congés maladie et des vacances payées, réduction des budgets militaires, démocratisation des entreprises et sociétés, parmi de nombreuses autres revendication - tout ceci présuppose la reconnaissance de cadres institutionnels existants, et est inévitablement adressé à leurs représentants.

La technique par laquelle les auteurs du manifeste traitent cette contradiction apparente est celle du schème récurrent de la concession. Celui-ci se construit par l'expression d'une résistance totale à un système ou un état des lieux existants, et un appel à son renversement fondamental, suivis par la déclaration que «tant que cela existe ", une réforme ou une réglementation s'imposent. C'était également le cas en ce qui concerne la Déclaration des Droits de l'Homme citée plus haut: une nouvelle Déclaration devrait être rédigée, mais tant que l'ancienne existe, "elle doit être appliquée à tous - aussi bien dans les pays riches que pauvres ». Ci-dessous, voici quelques exemples supplémentaires tirés de la liste des demandes formulées dans le manifeste : "Tant qu'ils existent, le FMI, la Banque Mondiale et le Comité de Bâle sur les Réglementations Bancaires doivent être radicalement démocratisés »; «Tant qu'ils existent, une réforme radicale et la démocratisation du système commercial mondial et de l'Organisation Mondiale du Commerce doivent intervenir »; "Tant qu'ils sont en vigueur, les systèmes électoraux doivent être aussi justes et représentatifs que possible »; « Tant que des inégalités sociales existent, les impositions à tous les niveaux doivent se plier au principe de solidarité».

Cette technique répétitive de concession permet aux auteurs du manifeste de transmettre un esprit et un concept révolutionnaire généraux, tout en proposant un programme quelque peu modéré. Il s'agit d'une technique typique de ce que Herbert W. Simons (1970: 10) nomme une stratégie rhétorique «intermédiaire» aspirant à concilier des stratégies modérées et militantes, dans un mouvement qui est polarisé à la base. Dans le cas du GlobalMay Manifesto, la polarisation est flagrante dès le départ en tant qu'aspiration à une validation universelle, apportant inévitablement des conditions, des comportements et des intérêts conflictuels, tandis que - comme mentionné précédemment - certains des membres s'opposent à l'idée même de tout programme établi. Le manifeste tente ainsi de jongler entre un désir de déclarations révolutionnaires romantiques, excitantes et attirantes, et la nécessité de procurer un 
plan concret, raisonnable, applicable et certainement moins excitant. Dans leurs propres termes sciemment oxymoriques de la première partie du manifeste, les auteurs proposent une combinaison d'action modérée et de ton combatif - une "rage empreinte de dignité $»^{26}$.

\section{Universalisme et politiques identitaires : le « nous » et sa déconstruction}

La tentative d'harmoniser des points conflictuels et de produire une unification dans une situation de polarisation immanente est visible - on l'a vu - à travers un autre conflit dominant dans le manifeste : celui qui oppose universalisme et individualité. Au cours des dernières décennies, les politiques identitaires ont investi d'énormes efforts dans la critique du discours libéral des droits universels. Les discours des genres et des minorités, des droits des animaux et des mouvements de protection des enfants, parmi d'autres, ont révélé les mécanismes de suppression inhérents au langage prétendument inclusif de l'universalisme, enraciné dans le discours politique libéral des Lumières du $18^{\mathrm{e}}$ siècle. Les politiques identitaires s'efforcent de révéler la grande variété des identités supprimées par le «nous » universel, et de démasquer l'illusion d'égalité qui implique des pratiques importantes d'exclusion ${ }^{27}$. Le succès remarquable des politiques identitaires ne se traduit pas seulement par la transformation des normes et régulations - ainsi que par sa forte pénétration dans la sphère académique, mais aussi, à l'évidence, dans le discours populaire et la rhétorique, où son expression ultime est celle du langage politiquement correct.

Par son appel à une démocratisation mondiale, le GlobalMay Manifesto utilise un discours d'universalisme nettement emprunté à l'ethos universel de la Déclaration des Droits de l'Homme française; mais parallèlement, il marque qu'il est conscient des sensibilités contemporaines liées au discours des politiques identitaires, et se confronte inévitablement à ses exigences telles qu'il se les imagine. Cette position est d'ailleurs clairement exposée dans la proposition de rédiger une nouvelle Déclaration des Droits de l'Homme adaptée au $21^{\mathrm{e}}$ siècle, mais elle s'exprime également par de nombreuses postures textuelles tout au long du manifeste. L'affrontement entre l'urgence de construire un collectif unifié universel par le biais du pronom «nous $»^{28}$ et le besoin de s'adresser à des identités variées qui décentralisent et déconstruisent les efforts d'unification, est net dans le texte - ce que je me propose de démontrer à présent.

L'effort investi pour construire un sentiment d'universalité s'exprime, surtout et avant tout, dans le désir de présenter le manifeste comme un texte anonyme qui n'est pas limité par un langage concret, comme s'il était écrit dans une langue universelle transgressant toutes les barrières culturelles. Le manifeste est accessible par un lien rédigé en plusieurs traductions, présentées par ordre alphabétique : allemand, anglais, chinois, espagnol et portugais ${ }^{29}$. De la sorte, l'anglais, qui n'est pas le premier de la liste, est présenté comme étant juste " une langue parmi d'autres ", l'occultant ainsi en tant que langue originelle du texte, ce qui estompe le fait que le manifeste a été rédigé principalement par des activistes anglo-américains ${ }^{30}$. Une technique similaire a été appliquée dans la rédaction du Manifeste Communiste, dans sa tentative d'édifier une classe ouvrière universelle. Au début du manifeste, Marx et Engels déclarent que le texte sera publié en " anglais, français, allemand, italien, flamand et danois ", plaçant l'allemand au milieu de la liste comme s'il ne s'agissait que d'une langue parmi d'autres, 
et non pas de la langue d'origine. Le Manifeste Communiste se présente ainsi comme un texte universel qui traverse les frontières des identités nationales et, de ce fait, sa première édition a été publiée sans la signature des auteurs, comme un pamphlet anonyme (Puchner 2006 : 23).

Cet ethos universel du GlobalMay Manifesto, indiqué par le «nous » récurrent, globalisant (qui ouvre pratiquement chaque paragraphe de la première partie) est subverti par deux procédés principaux. Le premier, direct, est celui qui consiste à insérer les politiques identitaires dans la liste des exigences du manifeste. L'une de ces exigences est que "les droits civiques, culturaux, politiques et économiques des minorités sexuelles, culturelles et ethniques doivent être pleinement reconnus ». Une autre exige le «respect complet des droits des enfants». Cependant, une autre pratique linguistique moins directe n'en est pas moins significative : l'ethos universel est subverti par des expressions qui, parsemées tout au long du texte, minent le sentiment de collectivité homogène. Ainsi, à maintes reprises, le «nous" se scinde en «nous, femmes et hommes", comme s'il s'agissait d'une mesure venant corriger le sujet prétendument universel de la Déclaration des Droits de l'Homme et du Citoyen et son héritage, qui dissimulaient une exclusivité masculine. Une autre expression récurrente est le «certains d'entre nous» restrictif (« Nous n'adressons pas nos exigences aux gouvernements [...] que certains d'entre nous considèrent comme étant illégitimes »; "Certains d'entre nous pensent qu'une nouvelle Déclaration Universelle des Droits de l'Homme [...] devrait être rédigée »), qui remplace le «nous » homogène et implique des divisions et différences internes. Le manifeste se présente également comme le représentant de «nos mouvements » au lieu d'éveiller le sentiment qu'il s'agit d'un seul mouvement unifié, ce qui le présente comme une structure pouvant englober la multiplicité.

De plus, outre l'ethos collectiviste construit par le manifeste à la fois dans ses principes et exigences explicites (qui glorifient le concept du «bien commun») et son ton général (qui célèbre une universalité unifiée imaginaire), les auteurs se distancient également de l'ethos communiste rigide en montrant l'importance du bonheur individuel et de l'accomplissement de soi individualiste. L'une de leurs demandes, par exemple, indique "Outre le pain, nous voulons des roses. Tout le monde a le droit de jouir de la culture, de prendre part à des activités créatrices et enrichissantes au service du progrès de l'humanité ». La première partie du manifeste se termine par l'illustration de l'harmonie souhaitée entre les intérêts collectifs et individuels : «un monde dans lequel toute femme, homme et enfant sera certain d'avoir le droit de rechercher librement le bonheur, aussi bien personnel que collectif ».

\section{Environnementalisme : d'un discours universel à un discours sur l'univers}

31 Dans l'une des parties du GlobalMay Manifesto qui soumettent des exigences, il est dit que "notre changement de mode de vie sera biologique/écologique ou ne sera pas ». Cette déclaration illustre la prépondérance du discours écologique dans la rhétorique de ce manifeste, et sa tendance à dénoncer le comportement écologique immoral comme un facteur primordial de la corruption générale de la société : « les générations futures sont condamnées à un héritage empoisonné dû aux crimes écologiques des riches et des puissants [...] sans considération pour le destin de la planète et de ses 
habitants». Il semble que dans la totalité du manifeste, les termes "écologie » et " planète » deviennent synonymes du mot « société ». Ainsi, dans sa liste de demandes, la première exigence indique : "L'économie doit être mise au service du bien-être du peuple, et doit soutenir et servir l'environnement, et non le bénéfice privé ». Le profit privé est mis ici en contraste non pas avec le profit de la communauté, mais avec celui de tout l'environnement naturel. Cinq des dix sous-sections de cette déclaration sont consacrées exclusivement à réglementer les normes écologiques, telles l'appel à la responsabilité des entreprises, la réduction de l'utilisation d'agrochimiques, l'agriculture durable et l'énergie renouvelable. "L'écocide (dégâts volontaires à l'environnement écologique, aux écosystèmes, à la biodiversité) ", déclarent les auteurs, " devrait être mondialement reconnu comme un crime de la plus grande magnitude ». En ne mentionnant même pas d'autres crimes collectifs contre l'humanité (tel le génocide, qui résonne dans «écocide»), les auteurs placent le comportement écologique au sommet de leur liste de préoccupations, faisant ainsi du manifeste non seulement un texte qui vise à être universel, c'est-à-dire valable dans le monde entier, mais aussi un texte qui voit dans la gestion des problèmes de l'univers sa mission la plus urgente.

32 La prédominance remarquable d'un langage écologique dans le texte reflète la capacité considérable de ce discours à s'infiltrer dans la sphère politique et à fonctionner comme un élément constitutif des manifestations socio-politiques au $21^{\mathrm{e}}$ siècle, en partie du moins grâce à la rhétorique militante de nombreux mouvements écologiques contemporains qui obéissent religieusement aux normes écologiques des organisations. Ainsi, face aux critiques éventuelles, les auteurs du manifeste sont indirectement astreints à fournir une vision du monde largement écologique - ce qui est devenu une évidence dans toute manifestation socio-politique mondiale ${ }^{31}$. On peut en déduire que, parallèlement à la sensibilité aux politiques identitaires, l'écologie est devenue partie intégrante de la doxa de l'activisme politique contemporain, au point que même lorsque le manifeste ne traite pas directement des problèmes écologiques, ce discours y est toujours présent - de manière implicite ou métaphorique, s'imposant comme une évidence. Ainsi, dans la partie initiale, les auteurs se présentent comme une communauté d' " habitants de cette planète " plutôt qu'en tant que citoyens du monde, et des motifs du même ordre sont constamment repris.

33 Tandis que les politiques identitaires contrarient l'impression d'avoir à faire à une collectivité homogène, le thème de l'écologie élargit la collectivité au-delà de ses limites - il implique que le «nous » ne se rapporte pas seulement à une association de citoyens mais à toute vie sur terre. En conséquence, en passant de l'universel à l'univers, le manifeste raconte l'histoire typique de peuples se soulevant contre d'anciens pouvoirs établis, pour lancer une mission de sauvetage.

\section{Conclusion : un texte à engagements multiples}

Le présent article a montré que les ambitions du GlobalMay Manifesto de construire une collectivité unifiée et d'atteindre à la validité universelle, dans la tentative de représenter un mouvement fondamentalement hostile à l'unification, entraînent une structure rhétorique hybride qui envoie des signaux rhétoriques à une large variété de destinataires potentiels. Ce manifeste est donc présenté ici non pas comme un texte qui s'engage pour réaliser un objectif bien défini ou construire un ethos cohérent, mais 
plutôt comme un texte fait d'engagements multiples et conflictuels envers divers organismes de la sphère sociale. Ce manifeste montre la façon dont confrontations et conflits entre activistes se traduisent non seulement dans la dynamique des rues, des squares et des réseaux sociaux, mais aussi dans l'espace imaginaire d'un langage déclaratif et programmatique. A l'inverse de la conception traditionnelle du manifeste comme genre monologique qui, essentiellement, « refuse le dialogue» (Lyon 1999: 9), le présent article souligne sa nature polyphonique potentielle.

Le ton général et la structure de ce manifeste exposent le modèle historique responsable de l'héritage génétique du genre du manifeste en général, et de celui du GlobalMay Manifesto en particulier : la Révolution française, et la Déclaration des Droits de l'Homme et du Citoyen. Apparaissant comme un «meme » tout prêt à travers ses tropes typiques et ses allusions, ce modèle constitue dans une large mesure l'ethos universaliste du texte. Cet héritage textuel est cependant sujet à des mutations constantes liées à des normes et des sensibilités culturelles en évolution, des exigences variables et les nouvelles technologies du $21^{\mathrm{e}}$ siècle.

En premier lieu, le ton universel-révolutionnaire unifié se confronte à de nouvelles possibilités technologiques en tout ce qui concerne la négociation collaborative, et à ce que l'on peut appeler une «cooptation numérique »: la possibilité de se joindre à la fabrication d'un texte programmatique grâce à une communication numérique en direct. Ces possibilités réorientent la fonction des auteurs du manifeste : ils passent de représentants d'un pouvoir définitif au statut d'initiateurs d'un débat. De plus, le ton révolutionnaire est confronté à la volonté de jouir d'une approbation publique large et effective, ce qui modère l'agressivité inhérente au discours de rupture et dirige le manifeste dans la voie d'une solution par les réformes. Enfin, l'ethos universel de la Déclaration française se confronte à des discours populaires qui imposent de nouvelles exigences à l'activisme contemporain. Alors que les politiques identitaires affaiblissent le sentiment d'une collectivité unifiée, le discours écologique élargit cette collectivité, et présente la protestation à travers un narratif écologique étendu. Dans un sens, ces deux discours s'ajoutent aux "vérités évidentes" bien connues de la Déclaration française. La puissante communauté révolutionnaire unifiée que le manifeste aspire à créer est ainsi constamment construite et déconstruite tout au long du texte.

Les topoï variés et contradictoires sur lesquels se fonde le manifeste révèlent qu'il s'agit d'un champ de tensions et de compromis entre des images de soi et des comportements concurrents, des exigences conflictuelles et des méthodes d'action civile différentes. Ce simple cas a ainsi fourni l'occasion de discuter de la nature feuilletée de la rhétorique du genre manifestaire et, plus spécifiquement, des défis rhétoriques que ce genre moderne doit relever face aux technologies et aux sensibilités sociales du $21^{\mathrm{e}}$ siècle.

\section{BIBLIOGRAPHY}

Austin, J. L. 1962. How to do Things with Words (Cambridge, MA : Harvard University Press) 
Abastado, Claude. 1980. « Introduction à L'analyse des Manifestes », Littérature 39, 3-11

Amossy, Ruth. 2002. « How to Do Things with Doxa : Toward an Analysis of Argumentation in Discourse », Poetics Today $23: 3,464-487$

Angenot, Marc. 1982. La parole pamphlétaire : contribution à la typologie des discours modernes (Paris : Payot)

Bowers, John W., Donovan J. Ochs \& Richard J. Jensen. 2010. The Rhetoric of Agitation and Control, $3^{\mathrm{e}}$ éd. (Long Grove, IL : Waveland)

Burger, Marcel. 2002. Les manifestes : paroles de combat. De Marx à Breton (Lausanne : Delachaux \& Niestlé)

Caws, Mary Ann, éd. 2001. Manifesto : A Century of Isms (Lincoln: University of Nebraska Press)

Demers, Jeanne \& Line Mc Murray.1986.L'enjeu du manifeste, le manifeste en jeu (Quebec : Le Préambule)

Glasius, Marlies \& Geoffrey Pleyers. 2013. « The Moment of 2011 : Democracy, Social Justice, Dignity ", Development and Change $44: 3,547-567$

Hjartarson, Benedikt. 2007. « Myth of Rupture : The Manifesto and the Concept of Avant-Garde ", Eysteinsson, Astradur \& Vivian Liska (eds), Modernism (Amsterdam : Benjamins), 93-173

Koolhaas, Rem. 1994 [1978].Delirious New York : A Retroactive Manifesto for Manhattan (New York : Monacelli Press)

Lyon, Janet.1999. Manifestos: Provocations of the Modern (Ithaca, NY : Cornell University Press)

Marcoccia, Michel. 1994. Le rôle de porte-parole dans le discours politique. Analyse socio-pragmatique, thèse de doctorat en sciences du langage, Université Lyon 2

Orkibi, Eithan. 2012.Les étudiants de France et la guerre d'Algérie : identité et expression collective de l'UNEF (Paris : Syllepse)

Orkibi, Eithan. 2013. «Critique et engagement dans la rhétorique des mouvements sociaux », Argumentation et Analyse du Discours 11 [en ligne : http://aad.revues.org/1559, consulté le 1 mars 2015]

O'Sullivan, Luke. 2012. «Democracy or Just Demo Crazy? A Critical Examination of the 2012

Global May Manifesto », Cuadernos de Pensamiento Politico 36, 77-102

Perloff, Marjorie. 2003. The Futurist Moment (Chicago \& London : University of Chicago Press)

Puchner, Martin. 2006. Poetry of the Revolution: Marx, Manifestos and the Avant-Gardes (Princeton, $\mathrm{NJ}$ : Princeton University Press)

Shifman, Limor. 2014. Memes in Digital Culture (Cambridge, MA : MIT Press)

Simons, Herbert W. 1971. «Requirements, Problems and Strategies : A theory of persuasion for social movements ", Quarterly Journal of Speech $56: 1,1-11$

Sinkey, Anne. 2009. The Rhetoric of the Manifesto, thèse de doctorat, Emory University

Somigli, Luca. 2003. Legitimizing the Artist : Manifesto Writing and European Modernism, 1885-1915

(Toronto : University of Toronto Press)

Winkiel, Laura. 2006. « The Rhetoric of violence : Avant-Garde Manifestos and the Myths of Racial community », Bru, Sacha \& Gunther Martens (éds), The Invention of Politics in the European AvantGarde (1906-1940) (Amsterdam : Rodopi), 65-90 
Yanoshevsky, Galia. 2009. «Three Decades of Writing on Manifesto : The Making of a Genre ", Poetics Today $30: 2,257-286$

\section{APPENDIXES}

\section{The "GlobalMay manifesto" of the International Occupy assembly}

We are living in a world controlled by forces incapable of giving freedom and dignity to the world's population. A world where we are told "there is no alternative" to the loss of rights gained through the long, hard struggles of our ancestors, and where success is defined in opposition to the most fundamental values of humanity, such as solidarity and mutual support. Moreover, anything that does not promote competitiveness, selfishness and greed is seen as dysfunctional.

But we have not remained silent! From Tunisia to Tahrir Square, Madrid to Reykjavik, New York to Brussels, people are rising up to denounce the status quo. Our effort states "enough!", and has begun to push changes forward, worldwide.

This is why we are uniting once again to make our voices heard all over the world this 12 May.

We condemn the current distribution of economic resources whereby only a tiny minority escape poverty and insecurity, and future generations are condemned to a poisoned legacy thanks to the environmental crimes of the rich and powerful.

"Democratic" political systems, where they exist, have been emptied of meaning, put to the service of those few interested in increasing the power of corporations and financial institutions.

The current crisis is not a natural accident; it was caused by the greed of those who would bring the world down, with the help of an economics that is no longer about management of the common good, but has become an ideology at the service of financial power.

We have awakened, and not just to complain! We aim to pinpoint the true causes of the crisis, and to propose alternatives.

The statement below does not speak on behalf of everyone in the global spring/ Occupy/Take the Square movements. It is an attempt by some inside the movements to reconcile statements written and endorsed in the different assemblies around the world. The process of writing the statement was consensus-based, open to all, and regularly announced on our international communications platforms. It was a hard and long process, full of compromises; this statement is offered to people's assemblies around the world for discussions, revisions and endorsements. It is a work in progress.

We do not make demands from governments, corporations or parliament members, which some of us see as illegitimate, unaccountable or corrupt. We speak to the people of the world, both inside and outside our movements.

We want another world, and such a world is possible: 
1. The economy must be put to the service of people's welfare, and to support and serve the environment, not private profit. We want a system where labour is appreciated by its social utility, not its financial or commercial profit. Therefore, we demand:

- Free and universal access to health, education from primary school through higher education and housing for all human beings. We reject outright the privatisation of public services management, and the use of these essential services for private profit.

- Full respect for children's rights, including free childcare for everyone.

- Retirement/pension so we may have dignity at all ages. Mandatory universal sick leave and holiday pay.

- Every human being should have access to an adequate income for their livelihood, so we ask for work or, alternatively, universal basic income guarantee.

- Corporations should be held accountable to their actions. For example, corporate subsidies and tax cuts should be done away with if said company outsources jobs to decrease salaries, violates the environment or the rights of workers.

- Apart from bread, we want roses. Everyone has the right to enjoy culture, participate in a creative and enriching leisure at the service of the progress of humankind.

Therefore, we demand the progressive reduction of working hours, without reducing income.

- Food sovereignty through sustainable farming should be promoted as an instrument of food security for the benefit of all. This should include an indefinite moratorium on the production and marketing of genetically modified organisms (GMOs) and immediate reduction of agrochemicals use.

- We demand policies that function under the understanding that our changing patterns of life should be organic/ecologic or should never be. These policies should be based on a simple rule: one should not spoil the balance of ecosystems for simple profit. Violations of this policy should be prosecuted around the world as an environmental crime, with severe sanctions for those convicted.

- Policies to promote the change from fossil fuels to renewable energy, through massive investment which should help to change the production model.

- We demand the creation of international environmental standards, mandatory for countries, companies, corporations, and individuals. Ecocide (wilful damage to the environment, ecosystems, biodiversity) should be internationally recognised as a crime of the greatest magnitude.

2. To achieve these objectives, we believe that the economy should be run democratically at all levels, from local to global. People must get democratic control over financial institutions, transnational corporations and their lobbies. To this end, we demand:

- Control and regulation of financial speculation by abolishing tax havens, and establishing a Financial Transaction Tax (FTT). As long as they exist, the IMF, World Bank and the Basel Committee on Banking Regulation must be radically democratised. Their duty from now on should be fostering economic development based on 
democratic decision making. Rich governments cannot have more votes because they are rich. International institutions must be controlled by the principle that each human is equal to all other humans - African, Argentinian or American; Greek or German.

- As long as they exist, radical reform and democratisation of the global trading system and the World Trade Organization must take place. Commercialisation of life and resources, as well as wage and trade dumping between countries must stop.

-We want democratic control of the global commons, defined as the natural resources and economic institutions essential for a proper economic management. These commons are: water, energy, air, telecommunications and a fair and stable economic system. In all these cases, decisions must be accountable to citizens and ensure their interests, not the interests of a small minority of financial elite.

- As long as social inequalities exist, taxation at all levels should maintain the principle of solidarity. Those who have more should contribute to maintain services for the collective welfare. Maximum income should be limited, and minimum income set to reduce the outrageous social divisions in our societies and its social political and economic effects.

- No more money to rescue banks. As long as debt exists, following the examples of Ecuador and Iceland, we demand a social audit of the debts owed by countries. Illegitimate debt owed to financial institutions should not be paid.

- An absolute end to fiscal austerity policies that only benefit a minority, and cause great suffering to the majority.

- As long as banks exist, separation of commercial and financial banks, avoiding banks that are "too big to fail".

- An end to the legal personhood of corporations. Companies cannot be elevated to the same level of rights as people. The public's right to protect workers, citizens and the environment should prevail over the protections of private property or investment.

3. We believe that political systems must be fully democratic. We therefore demand full democratisation of international institutions, and the elimination of the veto power of a few governments. We want a political system which really represent the variety and diversity of our societies:

- All decisions affecting all mankind should be taken in democratic forums like a participatory and direct UN parliamentary assembly or a UN people's assembly, not rich clubs such as $\mathrm{G} 20$ or G8.

- At all levels we ask for the development of a democracy that is as participatory as possible, including non representative direct democracy.

- As long as they are practised, electoral systems should be as fair and representative as possible, avoiding biases that distort the principle of proportionality.

- We call for the democratisation of access and management of media. These should serve to educate the public, as opposed to the creation of an artificial consensus about unjust policies.

-We ask for democracy in companies and corporations. Workers, despite wage level or gender, should have real decision-making power in the companies and corporations 
they work in. We want to promote co-operative companies and corporations, as real democratic economic institutions.

-Zero tolerance of corruption in economic policy. We must stop the excessive influence of big business in politics, which is today a major threat to true democracy.

- We demand complete freedom of expression, assembly and demonstration, as well as the cessation of attempts to censor the internet.

- We demand respect for privacy rights on and off the internet. Companies and the government should not engage in data mining.

-We believe that military spending is politically counterproductive to a society's advance, so we demand its reduction to a minimum.

- Ethnic, cultural and sexual minorities should have their civil, cultural, political and economic rights fully recognised.

- Some of us believe a new Universal Declaration of Human Rights, fit for the 21st century, written in a participatory, direct and democratic way, needs to be written. As long as the current Declaration of Human Rights defines our rights, it must be enforced in relation to all - in both rich and poor countries. Implementing institutions that force compliance and penalise violators need to be established, such as a global court to prosecute social, economic and environmental crimes perpetrated by governments, corporations and individuals. At all levels, local, national, regional and global, new constitutions for political institutions need to be considered, as in Iceland or in some Latin American countries. Justice and law must work for all, otherwise justice is not justice, and law is not law.

This is a worldwide global spring. We will be there and we will fight until we win. We will not stop being people. We are not numbers. We are free women and men. For a global spring!

For global democracy and social justice!

Take to the streets in May 2012!

Source : GlobalMay Manifesto in The Guardian : http://www.theguardian.com/

commentisfree/2012/may/11/occupy-globalmay-manifesto, consulté le 1 mars 2015

\section{NOTES}

1. «Activismes post 2010 » définis par Marlies Glasius et Geoffrey Pleyers (2013: 547-548) comme vague de protestations distinctes, rassemblées autour de valeurs partagées de démocratie, justice sociale et dignité, «s'étalant des révoltes arabes au mouvement Occupy, les Indignados et les manifestations anti-austérité en Europe, ainsi que les manifestations pro-démocratiques en Russie et au Mexique ».

2. En 1978, Rem Koolhaas doutait de la validité du genre « manifeste », en débutant son New-York délirant : un manifeste rétroactif pour Manhattan par la question : "Comment écrire un manifeste en une période qui en est dégoûtée ?». Sur la perception commune du manifeste en tant que genre «mort », voir Martin Puchner (2006 : 241-243). 
3. Ainsi que le montre Galia Yanoshevsky (2009), le terme "manifeste » a été élargi de manière significative dans la recherche et la critique durant ces dernières décennies, et est devenu un intitulé englobant une vaste gamme de textes tels qu'introductions, catalogues, interviews, et œuvres d'art. Il est aussi devenu un titre populaire pour des groupes de rock et des produits de consommation tels les cosmétiques (Isabelle Rossellini, Yves Saint-Laurent), ce qui l'écarte de son contexte politique originel en tant que document d'agitation et d'engagement civique.

4. Des proclamations publiques, ainsi que l'indiquent Bowers et al. (2010:22-29), sont essentielles à l'établissement de mouvements sociaux. Sans pétitions ni proclamations, le mouvement pourrait n'être perçu que comme un groupe d'individus décentralisés. Marc Angenot (1982: 61) distingue le manifeste comme représentant une collectivité, contrairement à d'autres pratiques proclamatoires, telles le pamphlet.

5. GlobalMay Manifesto visait à jouer un rôle dans les manifestations socio-politiques globales projetées pour Mai 2012. Catia Gregoratti (2014: 357), se basant sur des interviews et des conversations, prétend que le manifeste est le résultat d'échanges en ligne qui ont eu lieu de Janvier à Mai cette année-là.

6. Occupy est l'un des mouvements sociaux mondiaux les plus marquants dans la vague manifestaire post-2010, basé à l'origine sur la prise de pouvoir temporaire de lieux publics. Il débuta avec Occupy Wall Street à New-York en 2011, et s'étendit rapidement surtout à travers les États-Unis et l'Europe. Aujourd'hui, le terme «occupy » est fréquemment utilisé pour qualifier toute agitation sociale mondiale contemporaine (par exemple, la page Wikipedia sur le mouvement considère les manifestations en Israël, en Turquie, en Afrique du Sud et autres, qui n'étaient pas à l'origine qualifiées "Occupy ", sous cette appellation.)

7. Voir cette série de débats sur le manifeste, dont la plupart l'attaquent violemment: http:// anticapitalists.org/tag/occupy-roundtable/, consulté le 1 mars 2015.

8. Voir l'accusation de manque d'agenda dans: http://www.globalpost.com/dispatch/news/ regions/americas/united-states/111101/occupy-americas-next-move, consulté le 1 mars 2015.

9. The Global Occupy Manifesto : A Demand to be Oppressed : http://anticapitalists.org/2012/06/18/ the-global-occupy-manifesto-a-demand-to-be-oppressed/

10. Occupy : Does it Really Need a Program ? : http://anticapitalists.org/2012/05/22/occupy-does-itreally-need-a-programme, consulté le 1 mars 2015.

11. Catia Gregoratti (2014) critique le flou créé par l'utilisation du terme « démocratie » dans des manifestes mondiaux contemporains, tels que The Manifesto for Global Democracy, GlobalMay Manifesto et The Peoples Petition. Elle critique le caractère compromettant de ces textes, qui supprime le potentiel du travail de terrain. Ces manifestes, affirme Gregoratti, ont été formulés surtout par des intellectuels privilégiés et ne reflètent pas la dynamique de la résistance populaire. Luke O'Sullivan (2014) critique l'échec du manifeste à proposer une vue mondiale cohérente, tout en appréciant cependant sa colère authentique.

12. Le concept du manifeste en tant qu'expression performative selon la terminologie de J. L. Austin (1962) est partagé par la communauté des chercheurs sur le manifeste, en commençant par les premières recherches de Claude Abastado (1980), en passant par les études typologiques de Jeanne Demers et Line Mc Murray (1986), jusqu'aux plus récentes recherches de Luca Somigli (2003) et de Martin Puchner. Puchner (2006 : 24-26) prétend cependant que n'ayant pas l'autorité antérieure nécessaire pour accomplir et satisfaire leurs actes de paroles, les créateurs de manifestes présentent un "théâtre d'autorité »- ils prétendent avoir la possibilité de réaliser leurs volontés à l'aide d'une gestuelle textuelle de puissance et de pouvoir.

13. Voir Eithan Orkibi (2013) pour une revue exhaustive du champ des études rhétoriques sur les mouvements sociaux.

14. GlobalMay Manifesto in People's Assemblies Network: http://www.peoplesassemblies.org/ 2012/05/may-12th-globalmay-statement/, consulté le 1 mars 2015. 
15. GlobalMay Manifesto in The Guardian: http://www.theguardian.com/commentisfree/2012/ may/11/occupy-globalmay-manifesto, consulté le 1 mars 2015.

16. Le manifeste est joint en annexe; toutes les citations qui suivront sont des extraits de cette version.

17. De nombreux manifestes s'achèvent par un appel à l'action, un motif de recrutement emprunté à la conclusion immortelle du Manifeste Communiste: «Travailleurs de tous les pays, unissez-vous!». Marc Angenot (1982:60) considère la qualité interpellative du manifeste comme une caractéristique fondamentale du genre dans le cadre de son débat sur La parole pamphlétaire.

18. Voir Ruth Amossy (2002) pour l'explication de la doxa en tant qu'élément constructif du discours. Contrairement à la critique idéologique qui dénonce la doxa comme étant la voix d'une idéologie hégémonique, Amossy (ibid : 465-467) la conçoit, à la suite de Chaïm Perelman, comme une structure contextuelle qui remplit des fonctions sociales fondamentales.

19. Au sujet de manifestes comme " mythes de rupture », voir Benedikt Hjartarson, 2011.

20. L'agressivité des manifestes a été soulignée par de nombreux chercheurs: Marcel Burger (2002) caractérise la rhétorique manifestaire comme étant fondamentalement combative. Bien qu'il se concentre surtout sur des manifestes d'avant-garde en les distinguant fortement comme un sous-genre à part (ibid,13), il présente les manifestes en général, ainsi que le suggère le titre de son étude, comme des "Paroles de combat». De même, Mary Ann Caws (2001: xx) décrit les manifestes comme des champs de bataille, Laura Winkiel $(2006: 67)$ dépeint les énonciations manifestaires comme des armes, et Marjorie Perloff (2003: 81) expose la conception des manifestes de F.T. Marinetti comme requérant intrinsèquement « de la violence et de la précision ».

21. Le terme "meme» a été inventé par Richard Dawkins (The Selfish Gene, 1976), pour décrire une conduite biologique répétitive, et a été largement emprunté par la recherche culturelle pour décrire la prolifération de certaines petites unités culturelles. Sur l'utilisation des memes en tant que méthode de participation politique, particulièrement dans le mouvement Occupy, voir Limor Shifman $2014: 119-150$.

22. Par exemple, au cours des manifestations de revendications sociales en Israël, en été 2011, tous ces symboles récurrents du meme Révolution française ont été utilisés, y compris l'installation d'une fausse guillotine sur le Boulevard Rothschild à Tel-Aviv, ainsi que la déclaration «vive la république des tentes!» dans Revolution Song-Book - Tent Poetry, publié de façon indépendante par une coopérative d'organisations (Daka, Maayan, Erev-Rav, Etgar, GerilaTarbut) en août 2011.

23. Le mot « révolution » décrivait à l'origine un mouvement circulaire et répétitif, tel celui des étoiles (OED).

24. Un autre exemple est celui de l'édition du Manifeste Communiste réalisée après la mort de Marx en 1883, dans laquelle l'introduction élégiaque d'Engels situe le manifeste comme un texte d'héritage et de patrimoine (Puchner $2006: 35$ ).

25. L'adresse ci-dessous permettait aux lecteurs de participer à l'écriture collective du Cyberpunk Manifesto : http://www.cyberpunkreview.com/; consulté le 1 mars 2015.

26. Voir Eithan Orkibi (2012) pour un débat au sujet de la construction de l'ethos collectif d'étudiants manifestants français pendant la Guerre d'Algérie. Orkibi montre que l'Union Nationale des Étudiants de France (UNEF) construit l'image des manifestants en tant que citoyens établis et responsables, contribuant ainsi de manière significative à leur légitimation. Par contraste, dans le GlobalMay Manifesto, l'oscillation hésitante entre une stratégie combative et modérée est apparente.

27. Au sujet de la présentation de soi commune aux auteurs de manifestes utilisant le "nous » collectif, et la problématisation de la subjectivité universelle du point de vue du genre (au sens de Gender), voir Janet Lyon (1999) et Anne Sinkey (2009). 
28. Voir Michel Marcoccia (1994) pour une discussion extensive sur le rôle du porte-parole, et l'utilisation de pronoms collectifs dans le discours politique.

29. Un lien vers les traductions du manifeste: http://www.peoplesassemblies.org/2012/05/ may-12th-globalmay-statement/, consulté le 1 mars 2015.

30. Catia Gregoratti $(2014: 358)$ constate que le manifeste fut rédigé par un nombre limité d'activistes principalement américains, sans la participation ni même la connaissance de la plupart des groupes actifs internationaux de Occupy de l'époque.

31. De fait, bien que le manifeste fut vivement critiqué par certains, plusieurs écologistes furent satisfaits par sa vision «verte ». Voir, par exemple, l'affichage de «TreeHugger »: http:// www.treehugger.com/environmental-policy/occupy-globalmay-manifesto-lots-greenmovement-should-get-behind.html, consulté le 1 mars 2015.

\section{ABSTRACTS}

GlobalMay Manifesto, spread on the internet in May 2012, is a collaborative document representing the global Occupy movement, one of few wide-spread international manifestos calling for global democratization, following the global social upheavals of 2011. The manifesto's attempt to provide a unifying ethos for a fundamentally decentralized and polarized movement was highly criticized, mainly for its vagueness and inconsistency. As opposed to the general evaluative character of the manifesto's popular and academic readings, this article explores its instrumental strategies for constructing an imagined global collectivity while responding to a wide range of potential requirements and complaints. The hybrid rhetorical structure of the manifesto, its alternating ethos and immanent heteroglossia, are explained as attempts to reconcile clashing attitudes and self-images. This case provides an opportunity to discuss the multilayered nature of the manifesto genre's rhetoric, specifically the rhetorical challenges it faces in the $21^{\text {st }}$ centur.

Le GlobalMay Manifesto diffusé sur Internet en mai 2012, est un document collaboratif représentant le mouvement mondial Occupy (Occuper) - l'un des manifestes internationaux appelant à la démocratisation mondiale suite aux agitations sociales universelles de 2011. La volonté du manifeste de fournir un ethos unifiant à un mouvement essentiellement décentralisé et polarisé a été vivement critiquée, surtout pour son vague et son manque de cohérence. Contrairement au caractère évaluatif des lectures populaires et académiques du manifeste, le présent article explore les stratégies mises en œuvre pour construire une collectivité mondiale imaginaire, dans une situation où il importe de se confronter à une gamme étendue de besoins et de récriminations potentiels. Il montre que la structure rhétorique hybride du manifeste, son ethos inconsistant et son hétéroglossie immanente, dérivent en fait de la volonté de concilier des attitudes et des images de soi discordantes. Ce cas de figure offre l'occasion d'explorer la rhétorique stratifiée du genre manifestaire, et plus spécifiquement les défis rhétoriques qu'il doit confronter au $21^{\mathrm{e}}$ siècle. 
INDEX

Keywords: collectivism, genre, global protest, GlobalMay Manifesto, heteroglossia, manifesto, meme

Mots-clés: collectivisme, genre, GlobalMay Manifesto, hétéroglossie, manifestation mondiale, manifeste, meme

\section{AUTHORS}

NANA ARIEL

Université de Tel Aviv 